\title{
Utopies sensorielles, contemplation mystique et sentiment de plénitude
}

Sensorial Utopia, Mystical Contemplation and Feeling of Plenitude

Jean-Pierre Albert

\section{Q OpenEdition}

Édition électronique

URL : http://journals.openedition.org/mythos/594

DOI : $10.4000 /$ mythos.594

ISSN : 2037-7746

Éditeur

Salvatore Sciascia Editore

\section{Édition imprimée}

Date de publication : 1 décembre 2017

Pagination : 69-82

ISSN : $1972-2516$

\section{Référence électronique}

Jean-Pierre Albert, «Utopies sensorielles, contemplation mystique et sentiment de plénitude », Mythos [En ligne], 11 | 2017, mis en ligne le 24 septembre 2019, consulté le 28 septembre 2019. URL : http:// journals.openedition.org/mythos/594; DOI : 10.4000/mythos.594 


\section{Utopies sensorielles, contemplation mystique et sentiment de plénitude}

\section{Jean-Pierre Albert}

\section{Résumé}

Un même idéal de plénitude en relation avec l'accès à une réalité transcendante se rencontre dans la mystique néoplatonicienne et la poésie amoureuse de Baudelaire. Dans les deux cas, une situation d'intense polysensorialité est supposée permettre cette expérience. L'introduction de la notion d'utopie sensorielle vise à analyser ces situations, vécues ou imaginées, en référence aux ressources dont les humains disposent pour échapper au vécu habituel de la temporalité. Certains rituels sont une solution possible. L'imaginaire du paradis dans les religions du Livre confirme la part d'une expérience sensible heureuse dans ce dépassement de la condition humaine. En même temps, la présence dans la littérature de paradis factices marque l'ambivalence de cette voie des sens dans l'accès à une plénitude spirituelle.

\begin{abstract}
A common ideal of plenitude that gives access to a transcendent reality can be found in Neoplatonic mysticism and Baudelaire's love poetry. In both instances, an intense multisensorial situation is meant to enable this experience. This article/chapter will analyze such situations, whether truly lived or fantasized, thanks to the concept of sensorial utopia. It will highlight the resources human have at their disposal to escape the routine experience of temporality. Certain rituals may offer a solution. The imaginary world of paradise in the religions of the Book illustrates the possibility of a sensible joyful experience to overcome the human condition. At the same time, the presence of artificial paradises in literature highlights the ambiguity of this sensorial pathway to reach a spiritual plenitude.
\end{abstract}

\section{Abstract \\ Néoplatonisme • mystique • paradis • polysensorialité • utopie \\ Mots clefs \\ Neoplatonism • mystic • paradise • multi-sensoriality • utopia}

e point de départ de cette étude, conduite dans le cadre du projet collectif Synaesthesia, est le texte de Plotin, philosophe néoplatonicien du III ${ }^{e}$ siècle, cité ci-après. Le philosophe vient de désigner l'univers comme un " animal ", et ce qui précisément l'anime n'est autre qu'un principe spirituel dont le prototype est la conception platonicienne de l'intelligible. Pour évoquer cette réalité ontologiquement supérieure, il poursuit en écrivant :

C'est comme un courant venu d'une source unique : il n'est pas comparable à un souffle ou une chaleur mais plutôt à une qualité unique qui possède et conserve en elle toutes les autres, à une douceur qui serait en même temps odeur, en qui la saveur du vin s'unirait à toutes les autres saveurs, et toutes les couleurs ; elle a toutes les qualités perçues par le tact et aussi toutes celles qui sont perçues par l'oreille, parce qu'elle est toute harmonie et tout rythme ${ }^{1}$.

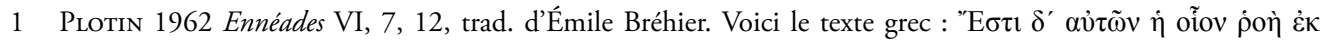

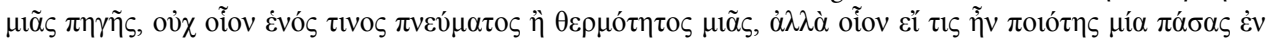

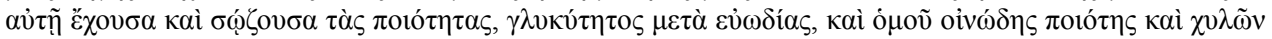

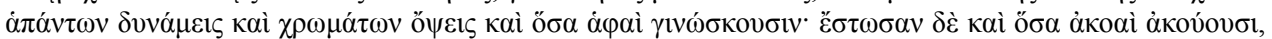

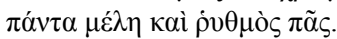


Il peut sembler paradoxal que, pour dire une entité située au-delà du sensible, Plotin développe une comparaison entre la saisie, purement intellectuelle sans doute, de ce principe spirituel et une expérience sensorielle imaginaire : une synthèse du meilleur de ce que chaque sens peut offrir. Plus précisément, c'est une expérience mystique de l'Un ou du Divin qui trouve à se dire dans le langage de la polysensorialité, comme si une expérience sensorielle de cette nature permettait justement d'aller au-delà du sensible.

Ce texte de Plotin m'a fait penser à la strophe finale d'un poème des Fleurs du Mal, "Tout entière ". Il s'agit d'une sorte d'anti-blason du corps féminin, puisque le poète affirme ne pas vouloir choisir entre les charmes de l'aimée : c'est « tout entière » qu'elle le ravit. Et il conclut :
Ô métamorphose mystique
De tous mes sens fondus en un.
Son haleine fait la musique,
Comme sa voix fait le parfum !

Bien des choses séparent, assurément, la contemplation néoplatonicienne du ravissement amoureux. Mais Baudelaire introduit le terme de "mystique " pour évoquer son état affectif et retrouve, à seize siècles de distance, la corrélation entre un imaginaire des sensations et l'accès à une forme de transcendance. On ne s'étonne guère, en même temps, que l'auteur de "Correspondances " (manifeste d'une poétique des synesthésies à l'égal du célèbre sonnet des voyelles de Rimbaud) se plaise à évoquer en ces termes des moments de plénitude amoureuse.

Que faire de ces références disparates ? Comment mettre ensemble Plotin et Baudelaire ? Mystique et polysensorialité ? Pour tenter d'y parvenir, je développerai dans mon analyse la notion d'utopie sensorielle, qui permettra d'articuler expériences sensibles et imaginaire, expressions approchées d'un ineffable et visée d'un absolu : autant d'éléments qui, bien que saisis dans cette première approche à travers quelques indices seulement, nous conduiront à aborder sous un angle nouveau de grandes traditions métaphysiques et religieuses. L'effet attendu du recours à la notion d'utopie est, en premier lieu, de suspendre la question de savoir si le sentiment de plénitude évoqué dans les textes renvoie à une expérience réellement vécue. Cela amène aussi à réfléchir sur la capacité de certaines situations - rituelles au premier chef - à introduire dans l'expérience des personnes une approximation de ce qui se donnait d'abord comme un idéal inaccessible : certains rituels, et plus précisément leur atmosphère sensorielle, ne seraient-ils pas, pour ceux qui les créent ou ceux qui les vivent, un moyen de réaliser l'utopie?

\section{Quelques préalables}

Bonheur et temporalité

Il y a lieu de penser que la difficulté qu'ont les humains à accéder à un sentiment de plénitude tient dans une large mesure à la forme temporelle de leur existence. J'entends ici par sentiment de plénitude un état affectif de pleine satisfaction subjective en relation avec un objet - Dieu, l'être aimé... Le terme d'objet est à vrai dire mal venu car ce qui se produit dans l'expérience mystique est plutôt un dépassement de la relation sujet-objet, que traduit par exemple l'idéal de fusion en Dieu. Il semble que de tels états dépassent la forme temporelle de l'existence, marquée en particulier par la tension du désir, et que la personne entre dans une sorte d'éternité. 
Échapper à la tyrannie du temps, c'est-à-dire avant tout à la projection dans le futur et à la crainte de la mort, s'exercer à vivre pleinement dans l'instant sont des thèmes majeurs des philosophies antiques, en particulier du stoïcisme et de l'épicurisme $e^{3}$. Dans cette dernière philosophie, la concentration heureuse sur l'instant suppose que l'on échappe à la pression toujours renaissante du besoin. Il ne saurait y avoir de plaisir là ou existe un sensation de manque, et c'est ce qui explique la relative austérité du mode de vie épicurien : une personne ressentira d'autant moins d'insatisfaction qu'elle aura soin de restreindre la gamme des besoins à satisfaire aux désirs naturels nécessaire ${ }^{4}$, auxquels peuvent se joindre sans trop de risque des désirs naturels non nécessaires, comme le plaisir pris au parfum d'une fleur. Chez les stoïciens également, il s'agit de vaincre le flux du temps en vivant chaque instant en harmonie avec l'ordre du monde. L'ataraxie est précisément ce vécu de l'instant hors des intérêts qui nous ancrent dans le tourbillon des événements. Telle est la voie du bonheur. Mais il y a lieu de douter qu'une telle expérience soit possible jusqu'au bout. Le bonheur, comme vécu d'une plénitude dans l'immanence, est-il à notre portée ? Selon Kant, "Le bonheur est un idéal de l'imagination et non de la raison "; "L'homme ne peut se faire un concept défini et sûr de cette somme de satisfaction à donner à toutes [ses inclinations] qu'il nomme le bonheur ${ }^{5}$. Cet idéal ne peut par conséquent pas devenir le but d'une existence, pour des raisons morales mais aussi parce qu'il est inatteignable.

On peut conclure de ce qui précède que la forme même de la subjectivité humaine tend à interdire toute échappée au-delà de l'ordre du manque, du désir, du souci, du projet. Le domaine des sensations lui-même ne peut s'en abstraire : l'acuité des sens s'estompe avec la durée - par exemple on cesse vite de sentir un parfum ou d'entendre un bruit continu. Accéder à une plénitude sensorielle supposerait donc la neutralisation de la structure temporelle qui marque la sensorialité elle-même. Ajoutons que cette plénitude est inséparable d'un certain plaisir, d'une satisfaction, voire d'une satiété qui marque justement l'épuisement du désir, ou la jouissance de l'objet vers lequel il tendait. Or la coloration positive de cette expérience supposée conduit à préciser la gamme des sensations les mieux à même de la produire. Quels sont donc, pour chaque sens, les objets les plus favorables ? Et ne faut-il pas en outre que les apports de chacun se rejoignent dans l'épaisseur d'une expérience polysensorielle ?

\section{Polysensorialité, optima sensibles et saturation sensorielle}

Revenons au texte de Plotin. Pour lui, la saisie de l'Intelligible est comparable à une sensation totalisante qui semble postulée plutôt que vécue. Plotin ne se contente pas d'énumérer les différents registres sensoriels dont il imagine la fusion (" une douceur qui serait en même temps odeur "), il met en avant pour ceux qu'il mentionne des exemples de sensations privilégiées : la douceur pour le tact, le vin pour le goût, l'harmonie et le rythme pour l'ouïe et les couleurs pour la vision. Le principe général de ces choix est qu’à chaque sens correspondent des objets suscitant un plaisir optimal : pour l'ouïe, c'est la musique, pour l'odorat le parfum, pour la vue la lumière et la couleur, etc. Il est aisé d'enrichir cette liste. Le plaisir du goût, par exemple, illustré dans le texte de Plotin par le vin, appelle souvent l'évocation du lait et du miel ; la « douceur " peut être tiédeur printanière ou caresse d'un tissu soyeux... Je propose de désigner

3 Sur les convergences entre les sagesses antiques, voir НADOT 1995.

4 Cette distinction est faite par Épicure dans sa Lettre à Ménécée, ÉpICure 1982, 77.

5 Kant 1978, 132, 98. 
l'expérience de ces objets privilégiés, correspondant à chaque gamme de sensations, comme des optima sensoriels. Il n'est guère douteux que leur désignation renvoie à une expérience possible, de même, jusqu’à un certain point, que leur rencontre synesthésique ( Il est des parfums frais comme des chairs d'enfants, / Doux comme les hautbois, verts comme les prairies [...] ») ou du moins polysensorielle. Mais cela appelle quelques précisions.

On peut tout d'abord se demander si la liste des optima sensoriels relève de codes culturels plus ou moins arbitraires ou si elle traduit un ensemble de contraintes liées à la nature de nos organes de sens et à nos capacités cognitives. En vérité, les deux propositions n'ont rien d'incompatible. Si les sources examinées plus loin présentent de fortes convergences, cela tient en partie à la relative proximité des cultures d'où elles émanent. Une approche plus fine permettrait de repérer des variations dans le temps et l'espace : on connait ainsi l'histoire des préférences en matière de parfums, par exemple le discrédit croissant des parfums musqués en Europe depuis le XVIII ${ }^{e}$ siècle et le triomphe de la jonquille ${ }^{6}$. Il reste que la catégorie " parfum ", ou le couple parfum/puanteur, a une validité transculturelle et renvoie probablement à des données biologiques. Il en va de même pour la catégorie "musique ", même si celle-ci offre des réalisations locales plus variées que celle de "parfum ». Dans les deux cas, du moins, les usages qui en sont faits, en particulier dans le domaine des rituels, laissent apparaitre des équivalences fonctionnelles qui concernent également leur utilisation conjointe.

Cela conduit à préciser un second point. Les références à Plotin et à Baudelaire présentées jusqu'ici évoquent des cas de polysensorialité ou de fusion des sensations qui font accéder à une réalité transcendante à travers une expérience mystique ou un sentiment de plénitude. Comment comprendre cette liaison ? Chaque homme en effet, sauf exception pathologique, est doté de registres sensoriels toujours en éveil dans sa vie ordinaire, à défaut de faire tous, ensemble et à chaque instant, l'objet d'une conscience explicite. Mais si n'importe quelle situation vécue est une occasion d'éprouver des sensations émanant de plusieurs organes des sens, ces derniers sont dans la plupart des cas inégalement sollicités : il est rare que, même pour un instant, nous soyons soumis à des stimuli assez saillants pour avoir simultanément conscience d'odeurs, goûts, couleurs, sons, impressions tactiles ou kinesthésiques qui concourraient tous à la qualité singulière de notre vécu du moment. Cela, toutefois, a d'autant plus de chances de se produire que les sensations concernées ont des propriétés spécifiques. Nous avons déjà rencontré un élément essentiel de cette spécificité à travers la notion d'optima sensoriels. Leur coprésence doit avoir pour effet d'élever les sensations au-dessus de la grisaille d'une polysensorialité ordinaire, aussi large soit-elle. Or cette coprésence des optima, le plus souvent, relève de l'artifice : celui du rituel ou de la mise en scène d'une intimité amoureuse elle aussi appareillée de parfums, bijoux, soieries, etc. Mais cela ne suffit pas encore. Je suggère que le pouvoir de la polysensorialité nécessite également un phénomène de saturation : couverture aussi complète que possible des registres sensibles et intensité des stimuli. L'expérience ethnographique des rituels confirme cette impression. La ritualité est en effet difficilement séparable de l'usage de musiques, parfums, mouvements rythmés, costumes bariolés, etc. dont l'effet conjugué engendre ou du moins favorise la transe ou l'extase, donc l'expérience d'un au-delà des réalités ordinaires. Quoi qu'il en soit de la réalité ou de la fréquence de ces états de conscience modifiée, ils sont tenus pour possibles et même recherchés. Je reviendrai plus loin sur le lien entre les rituels et les expériences mystiques. Il convient à présent de formuler quelques hypothèses 
sur les rapports entre expérience sensorielle et sentiment de plénitude tels qu'ils apparaissent dans le registre des représentations.

\section{Pour introduire les utopies sensorielles}

Des formes de plénitude ont pu être vécues dans les rituels ou des expériences mystiques avant d'influencer les descriptions idéalisées d'atmosphères à même de les soutenir. Si l'on se tourne vers les traditions mystiques présentes dans le néoplatonisme et les religions du Livre, on rencontre des modèles de contemplation qui se font écho sur un point essentiel : ils tracent des parcours qui, à travers le sensible, peuvent élever jusqu'au divin. Ces constructions reposent, inégalement selon les auteurs, sur des expériences effectives et des spéculations théologiques. On peut y reconnaître en outre une polarité entre la proposition d'exercices spirituels, que je n'examinerai pas ici, et le récit de contacts avec le divin : en contexte chrétien, visions et révélations privées, et plus encore relations corporelles avec Jésus présentées comme pourvoyeuses d'une ineffable félicité7.

Ces expériences de rencontre du divin font écho aux textes sacrés juifs, chrétiens et musulmans décrivant des lieux et des moments où des humains, morts ou vivants, sont supposés connaître une parfaite béatitude - il s'agit en l'occurrence d'images de paradis, terrestre ou céleste. Or ces descriptions accordent une place significative à des ambiances sensorielles, y compris quand elles concernent des humains devenus de purs esprits. Je propose d'appeler utopies sensorielles les récits ou descriptions porteurs de ces caractéristiques. Le terme d'utopie est pris dans son sens habituel - issu de l'ouvrage éponyme de Thomas More ${ }^{8}$ - de description d'un état idéal comme déjà réalisé. Sans doute le genre littéraire de l'utopie est-il toujours peu ou prou riche de notations sensorielles, dès lors que l'idéal décrit concerne la vie d'êtres humains connaissant dans la société où ils vivent un parfait bonheur. J'assume une extension de la notion permettant de rendre compte non seulement de sociétés idéales, mais aussi de situations dans lesquelles un sentiment de plénitude ou de béatitude absolue, tenu d'ordinaire pour inaccessible aux humains, est présenté comme effectivement vécu. Comme de telles expériences se heurtent au rapport de l'existence humaine à la temporalité, je serai attentif aux manières dont les descriptions utopiques portent la marque de cette difficulté, ou l'expriment à travers des thématiques particulières.

\section{Vers le spirituel à travers le sensible}

J'ai avoué en introduction mon étonnement initial devant le texte de Plotin : comment évoquer le suprasensible en comparant sa contemplation à une expérience sensible, imaginaire certes, mais bien ancrée dans le fonctionnement ordinaire de notre sensibilité ? De fait, la pensée de Platon, en dépit d'affirmations qui durcissent parfois sa métaphysique dualiste, laissait déjà une place au sensible dans l'élévation vers l'intelligible. Dans Le Banquet, l'amour humain et l'amour de la beauté sont présentés comme des médiations possibles, sinon nécessaires. Chez les néoplatoniciens, l'idée de la procession, c'est-à-dire de l'immanence de l'intelligible dans le sensible et l'activité de connaissance qui en résulte, confirme cette orientation. Et cette connaissance, acquise à travers une attitude contemplative, relève de la mystique autant ou plus que d'une métaphysique philosophique.

7 Voir par exemple Albert 1997.

8 L'ouvrage paraît en 1516, comprenant une critique de la situation sociale de l'Angleterre contemporaine et la vision de la société idéale de l'Ile d'Utopie. 
Pour les auteurs chrétiens inspirés par le néoplatonisme, dont l'énigmatique Pseudo-Denys ${ }^{9}$, le passage par le sensible pour accéder au divin devient une nécessité. Du fait de sa nature à la fois charnelle et spirituelle, l'homme ne peut accéder directement au divin, il ne peut l'approcher intellectuellement que par des analogies avec le monde sensible. Denys écrit ainsi dans sa Hiérarchie céleste :

[...] notre esprit ne saurait se hausser à cette imitation et contemplation immatérielle des hiérarchies célestes à moins d'y être conduit par des images matérielles convenant à sa nature, en sorte qu'il considère les beautés apparentes comme des copies de la beauté inapparente, les parfums sensibles comme des figures de la diffusion intelligible et les lumières matérielles comme des images du don de lumière immatériel, en sorte que les détours dont usent les enseignements sacrés représentent pour lui la plénitude de contemplation selon l'esprit, l'ordre des dispositions d'ici-bas l'habitus adapté aux réalités divines et ordonné, la réception de la très sainte Eucharistie la participation à Jésus, de façon qu'il sache que tous les autres dons, transmis aux essences célestes sur un mode supra - mondain, nous ont été livrés, à nous, en forme de symboles ${ }^{10}$.

Toute réalité terrestre peut, avec plus ou moins de succès selon sa place dans la hiérarchie des êtres, servir de tremplin à l'élévation vers le divin pour celui qui met en œuvre la forme de méditation dite anagogique. L'auteur ouvre son élucidation du recours à des « images matérielles » par trois exemples de registres sensoriels présents dans la liturgie des offices. Bien plus, les références au parfum et à la lumière relèvent des optima sensoriels. Réciproquement, les réalités célestes sont évoquées dans le langage du sensible : beauté, suavité des parfums, illumination. La théologie mystique de Denys suggère ainsi un lien entre une expérience de contemplation et un contexte rituel - explicitement dans la référence à l'eucharistie - qui est supposé favoriser l'élévation de l'esprit vers le divin. Rien dans son œuvre ne suggère toutefois que la voie du rite serait toujours requise pour ce type d'expérience. Et pourtant, il y a là une suggestion du plus grand intérêt, dont on va voir à présent l'écho qu'elle a trouvé, au XII siècle, dans le grand œuvre de l'Abbé Suger : la reconstruction de l'église abbatiale de Saint-Denis.

\section{Le Saint-Denis de Suger : un dispositif anagogique?}

C'est chez un lecteur assidu du Pseudo-Denys que le langage liturgique et architectural acquiert son plus grand potentiel anagogique. Le bâtisseur de la nouvelle église fit graver audessus de sa porte cette devise typiquement dionysienne : "L'esprit aveugle surgit vers la vérité par ce qui est matériel et, voyant la lumière, il ressuscite de sa submersion antérieure $» .{ }^{11}$ Cette formule évoque assurément pour Suger une théologie de la lumière dont Georges Duby a magnifiquement mis au jour les implications concrètes dans les choix esthétiques de l'abbé. Deux champs d'application d'une esthétique/une théologie de la lumière sont à retenir : à travers les grandes verrières de ce qui sera le prototype de la cathédrale gothique, le nouveau sanctuaire est traversé de flux lumineux ignorés des édifices romans, des flux magnifiés encore par la riche

9 Il s'agit en fait de l'auteur anonyme de traités théologiques attribués au Moyen-Âge au Denys converti à Athènes par saint Paul (Actes des Apôtres, 17, 34). Les textes ont été écrits en grec autour de l'an 500, probablement par un moine syrien.

10 Pseudo-Denys 1958, 72-73..

11 Cité dans Duby 1966, 14. Je m’appuie sur les pages 13-23 du livre. 
coloration des vitraux ; concernant le mobilier liturgique, Suger le pare d'une débauche d'ors et de pierreries. Citons après tant d'autres la formule où l'illustre abbé exprime le mieux le lien entre sa méditation mystique et ses choix esthétiques :

Lorsque, tout pénétré par l'enchantement de la beauté de la maison de Dieu, le charme des gemmes multicolores m'a conduit à réfléchir, transposant ce qui est matériel en ce qui est immatériel, sur la diversité des vertus sacrées, alors il me semble que je me vois moi-même résider comme en réalité en quelque étrange région de l'univers qui n'existe antérieurement ni dans le limon de la terre ni dans la pureté du ciel, et que, par la grâce de Dieu, je puis être transporté d'ici-bas dans le monde plus élevé de manière anagogique $»{ }^{12}$

Dans la ligne des techniques de méditation héritées du Pseudo-Denys, Suger trouve ainsi dans la contemplation des gemmes les chemins du ciel. Par leurs qualités sensibles - couleur, transparence et lumière - et leur place élevée dans la hiérarchie des êtres ${ }^{13}$, celles-ci semblent appartenir moins au monde de la matière qu'à celui de l'esprit. Elles sont, comme les aromates avec qui elles cohabitent dans le paradis terrestre des légendes, un point de passage entre ciel et terre, une manifestation terrestre de la présence du divin. Et c'est encore à une autre lumière, celle qui descend des vitraux, que Suger demande d'instituer le climat mystique du sanctuaire. Lordonnance des sensations visuelles et de leur pouvoir évocateur ne passe pas ici par la fabrication d'une image, ou la mise en scène d'un spectacle. Il s'agit plutôt de constituer une atmosphère, où le jeu du clair et de l'obscur, la focalisation conjointe de la lumière et du sacré dans les ors d'un ciboire ou les joyaux d'un reliquaire s'offrent comme une expérience globale, inséparable de la musique de l'office et du parfum de l'encens. N'est-ce pas là une heureuse approximation de l'expérience sensible totale que décrivait Plotin ? Une chose est certaine à mes yeux : la liturgie dans l'église de Saint-Denis entre dans la rubrique des utopies sensorielles. Il fallait ce contexte saturé de sensations heureuses pour que Suger emprunte avec succès les chemins anagogiques qu'il évoque dans le texte cité plus haut. Mais le registre du religieux n'a pas le monopole de ces expériences. Nous allons voir à présent comment la polysensorialité peut nourrir une idéalisation de l'être aimé qui n'est pas sans analogie avec la contemplation des mystiques.

\section{Les Fleurs du mal : une mystique de l'amour}

L'amour est un des thèmes dominants du recueil de Baudelaire mais, comme son titre le suggère, il en offre, comme de beaucoup d'autres aspects de la condition humaine, une image souvent noire, voire désespérée. En contrepoint, des poèmes répartis sur l'ensemble du recueil donnent de l'amour une version heureuse, apaisée, dans laquelle la femme aimée fait l'objet d'une vénération teintée de mysticisme. Ce sont ces poèmes-là qui vont à présent nous intéresser. Une étude, réalisée il y a plusieurs années, sur la place des parfums, dans les Fleurs du Mal, m'avait permis d'établir un corpus d'une dizaine de poèmes dont je m'aperçois aujourd'hui qu'ils associent presque tous l'odorat à d'autres registres sensoriels ${ }^{14}$. Et cela dans

12 Trad. de G. Duby, dans Duby 1966, 16.

13 Albert 1990, 76-80.

14 Il s'agit, dans leur ordre d'apparition dans le recueil, des poèmes intitulés "Correspondances ", "La Vie antérieure ", " Parfum exotique ", "La chevelure », "Le parfum », " Tout entière ", " L'invitation au voyage », " Moesta et errabunda ", "Les bijoux ", ce dernier faisant partie des pièces condamnées à l'issue du procès de 1857. 
une logique des optima : les perceptions associées au parfum sont la musique, la douceur des tissus (" mousseline ou velours » dans « Le parfum »), les couleurs du couchant, le lustre d'un meuble ancien... Nombreuses sont, en outre, les notations sinon de synesthésies, du moins de recouvrements de sensations relevant de registres différents :

Comme d'autres esprits voguent sur la musique,

Le mien, ô mon amour ! nage sur ton parfum. [...]

Un port retentissant où mon âme peut boire

À grands flots le parfum, le son et la couleur.

( L La chevelure »)

Les houles, en roulant les images des cieux,

Mêlaient d'une façon solennelle et mystique

Les tout-puissants accords de leur riche musique

Aux couleurs du couchant reflété par mes yeux.

( La Vie antérieure »)

Guidé par ton odeur vers de charmants climats, Je vois un port rempli de voiles et de mâts

Encor tout fatigués par la vague marine,

Pendant que le parfum des verts tamariniers,

Qui circule dans l'air et m'enfle la narine,

Se mêle dans mon âme au chant des mariniers.

(«Parfum exotique »)

À défaut de comporter des références au parfum, le poème "Les bijoux » offre enfin un magnifique exemple d'association de registres sensibles :

Quand il jette en dansant son bruit vif et moqueur,

Ce monde rayonnant de métal et de pierre

Me ravit en extase, et j'aime à la fureur

Les choses où le son se mêle à la lumière ${ }^{15}$.

Tous ces poèmes, sauf «La Vie antérieure ", sont hantés par la figure d'une femme aimée dont l'évocation est inséparable d'atmosphères saturées de sensations heureuses. Cela se jouant sur deux tableaux : celui de l'intimité amoureuse et érotisée de la chambre des amants ; celui des paysages qu'évoquent les sensations éprouvées en présence de l'aimée, dont le meilleur exemple est "L'invitation au voyage ". En d'autres termes, il semble que ces ensembles sensoriels soient la condition de l'accès à la femme aimée, dont l'adoration est teintée de mysticisme. La richesse des impressions sensibles qui se conjuguent semble ainsi produire à la fois des "moments d'éternité » et une absorption du sujet par l'objet de sa vénération. Faut-il se demander si le poète est sincère quand il décrit ces états d'âmes ? Considérer l'atmosphère sensible qu'il associe au bonheur amoureux comme une utopie sensorielle permet de laisser la question en suspens. Et il n'est que plus révélateur de l'importance des représentations et

15 Tous les textes sont cités à partir de Baudelaire 1961. 
pratiques de la sensorialité qu'elles se rencontrent à la fois dans la sphère du religieux et celle de l'amour profane. En abordant dans ce qui suit les descriptions des paradis, c'est de nouveau la question de cette continuité qui va se poser, question qui rejoint celle de la valeur du registre sensible par rapport à l'esprit ou au divin.

\section{Les paradis et leurs doubles}

Les descriptions bibliques du paradis, terrestre et céleste excèdent de multiples manières la seule perspective des utopies sensorielles. Cela est surtout vrai des deux textes de l'Apocalypse : liturgie céleste du ch. IV et évocation de la Jérusalem céleste au ch. XXI. Toutefois, je les aborderai brièvement afin d'en venir à une question qui, elle, concerne directement la valeur spirituelle accordée au domaine des sens, celle des paradis fictifs inspirés par le modèle du jardin d'Eden.

\section{Paradis liturgiques, paradis sanctuaires}

La vision du paradis du ch. IV de l'Apocalypse présente une liturgie d'adoration riche en fastes et trésors. Mais elle joue aussi sur l'effroi et la monstruosité - bruits de tonnerre, animaux chimériques. Rien ne permet d'établir un lien entre polysensorialité et béatitude des élus. En revanche, cet aspect redevient essentiel dans certaines visions du paradis, celles de Gertrude d'Helfta, par exemple, qui développe le texte de l'Apocalypse en imaginant des liturgies complexes et fastueuses ${ }^{16} \ldots$. Concernant le rapport à la temporalité, on notera que la liturgie d'adoration consiste en la répétition infinie d'une même séquence cérémonielle à laquelle on peut supposer que les élus participent comme spectateurs. À partir de notre expérience humaine du temps, nous imaginons sans peine l'insondable ennui qui nous saisirait si nous assistions à cette messe interminable... En fait, il est probable que le motif de la séquence indéfiniment réitérée vise à évoquer un au-delà du temps, donc un régime temporel compatible avec l'éternité de la béatitude.

La Jérusalem céleste, quant à elle, reprend du paradis terrestre, avec quelques modifications, la fontaine et l'arbre de vie. Elle constitue (si l'on laisse de côté, comme du reste l'ont fait l'iconographie et la symbolique chrétiennes, ses dimensions gigantesques) une sorte de cité-sanctuaire d'une totale perfection. Les murs qui l'entourent sont de pierres précieuses, le long inventaire qui en est fait évoquant une richesse sans limite et une beauté accomplies ${ }^{17}$. Ici encore, le rapport au vécu humain de la temporalité semble dépassé. Le monde contemporain de la Cité des derniers temps ignore la mer, figure de l'inconstance, comme elle-même ignore l'alternance du jour et de la nuit et les saisons. À la mesure des matériaux inaltérables dont elle est faite, elle appartient à l'éternité.

\section{Des paradis-jardins}

La description biblique du jardin d'Eden du chapitre II de la Genèse est plutôt laconique, mais elle fournit des éléments très suggestifs aux innombrables gloses et amplifications dont le Moyen Âge offre une large moisson. Ces textes dérivés brodent jusqu'à la démesure sur les expériences sensibles que réserve le paradis, et celles-ci sont souvent centrées sur les optima de chaque sens. Lisons, par exemple, la très célèbre Navigation de saint Brendan à la recherche du Paradis, un des exemples médiévaux les plus connus de voyages dans l'Autre Monde :

16 Par exemple une liturgie imageant l'intercession des saints : GerTrude D’helfTA 1978, 255.

17 Il s'agit des douze gemmes du pectoral d'Aaron, dont la liste a donné lieu à une abondante exégèse allégorique. 
Ils [les navigateurs] voient une terre très fertile en beaux bois et en prairies. Les prés, splendides et constamment en fleurs, y forment un jardin. Les fleurs sentent très bon, comme il convient à un endroit qu'habitent les saints, un lieu où les arbres et les fleurs font les délices de ceux qui les regardent, et où les fruits et les parfums sont d'une richesse inestimable. Ni ronces, ni chardons, ni orties n'y poussent à profusion ; il n'y a pas d'arbre ni d'herbe qui n'exhale une odeur suave. Les arbres sont continuellement chargés de fruits, et les fleurs toujours en plein épanouissement, sans tenir compte de la saison qui ne change pas ; c'est toujours l'été, et le temps reste doux. Les fruits sont toujours mûrs sur l'arbre. Les fleurs produisent sans cesse leur semence ; les bois sont toujours remplis de gibier, et toutes les rivières d'excellents poissons. Il y a des rivières où coule le lait. Cette abondance règne partout : les roselières exsudent le miel grâce à la rosée qui descend du ciel. Il n'y a pas de montagne qui ne soit d'or, pas de grosse pierre qui ne vaille un trésor. Le soleil ne cesse d'y briller de tout son éclat, aucun vent, aucun souffle ne vient remuer le moindre cheveu, aucun nuage dans le ciel ne masque la lumière du soleil. L'’habitant n'y souffrira aucun malheur, il ne connaîtra aucun orage, il sera à l'abri du chaud, du froid, de l'affliction, de la faim, de la soif, de la privation. Il aura tout ce qu'il souhaite, en abondance. Il est certain de ne jamais être privé de ce qu'il désire le plus; il l'aura toujours à sa disposition ${ }^{18}$.

Voilà donc un monde immuable et épargné par les affres du désir et du besoin. Selon la théologie de l'auteur, ce paradis terrestre semble être en fait un espace métaphysique où les élus, après leur mort, attendent le jugement dernier. Mais, en même temps, il revient au seul registre des sensations de construire une image de la béatitude. Cela explique peut-être pourquoi les amplifications du texte biblique ont en commun de multiplier les notations sensorielles. En même temps, le monde ainsi évoqué se rapproche étrangement d'un " pays de Cocagne » beaucoup moins catholique...

\section{L'ambivalence du sensible}

Le jardin paradisiaque, dans ce qu'il a de terrestre, nous rappelle qu'il n'y a qu'un pas entre sensorialité et sensualité. Ses fastes sensoriels peuvent suggérer une version hédoniste de la béatitude et, du fait de cette séduction, faire l'objet de transpositions profanes ou de mystifications intéressées. Avant d'aborder ce dossier, il faut dire quelques mots d'un hapax iconographique qui offre une version saisissante de l'ambivalence de la sensualité : le triptyque du Jardin des Délices de Jérôme Bosch. Le nom qui lui est donné réfère en vérité au panneau central, dont le sujet exact est difficile à identifier. Il ne s'agit pas, en effet, comme dans d'autres triptyques du même auteur, d'une évocation plus ou moins satirique de la réalité de ce monde et des vices et violences dont il est le théâtre. D'ailleurs, il est en totale continuité stylistique et thématique avec le panneau de gauche qui représente le jardin d'Eden. Je suivrai ici l'interprétation que propose Hans Belting ${ }^{19}$. Selon lui, le panneau central est bien, lui aussi, une évocation de l'Eden de la Genèse - on y retrouve par exemple les quatre fleuves en arrière-plan. Belting note également une étrangeté dans les représentations d'Adam et Ève au paradis terrestre : il manque la scène du péché originel. Son hypothèse est que le panneau central évoque l'humanité telle qu'elle aurait vécu en l'absence de la faute. Qu'elle ait été destinée à « croître et multiplier " peut se conclure de l'antériorité de cette prescription divine sur la tentation. La foule

18 Saint Brendan sd. : 118-122.

19 Belting 2002. 
qui peuple le Jardin des Délices est donc bien une humanité virtuelle qui conserve l'innocence du couple originel : d'où la nudité de tous, mais aussi une sensualité que traduisent baisers, contacts, voire gestes plus explicitement érotiques ${ }^{20}$. Cela se mêlant à l'évocation d'autres plaisirs sensibles, avec les agréments du bain, la tiédeur d'un éternel printemps et, ce qui est le plus visible, les plaisirs de la bouche évoqués par des fruits géants : l'utopie (le terme est utilisé par Belting) du jardin est bien une utopie sensorielle. Mais les êtres que l'on voit agir ne sont pas tout à fait des hommes - ils n'ont pas la connaissance du bien et du mal - et il faut sans doute donner tout son sens à leur proximité des animaux, eux aussi très nombreux. On peut leur prêter également un autre rapport au temps que le nôtre : voués à l'immortalité, échappant au vieillissement, exempts de tout désir du fait de son immédiate satisfaction, ils connaissent une sorte de plénitude qui n'est plus accessible à l'humanité post-édénique, mais qui reste engluée dans le sensible.

Cette ambivalence du sensible se retrouve dans plusieurs reprises du modèle édénique dans la littérature courtoise et son iconographie, par exemple Le roman de la rose de Jean de Meung, qui oppose l'espace de l'amour courtois - le verger de déduit - au plus orthodoxe " parc de l'Agneau ». Et le premier, qui suscite de dangereuses illusions, "n'a pas d'autre réalité que l'accumulation de synesthésies euphorisantes $»^{21}$. La littérature médiévale offre en outre plusieurs exemples de faux paradis qu'un personnage malveillant destine à tromper les âmes naïves. Ainsi celui du “Vieux de la montagne »"22, évoqué par Marco Polo dans son récit de voyage. L'histoire est bien connue : un seigneur pervers assure son pouvoir grâce à la fidélité de jeunes hommes qu'il enlève et fait résider quelques temps dans un lieu qu'il leur désigne comme le (vrai) paradis. Marco Polo lui-même sait reconnaître dans cette mise en scène le modèle du paradis coranique, tel qu'il est décrit dans la sourate 56 comme un « jardin des délices » conjuguant les plaisirs des fruits et de l'ivresse au luxe du mobilier et au service des houris « aux yeux grands et beaux $»^{23}$. Le piège paradisiaque du Vieux de la montagne donne à ces plaisirs une extension encore plus merveilleuse :

Il habitait une très haute vallée entre deux très hautes montagnes ; il y avait fait faire le plus vaste et superbe jardin qui jamais fut vu. Il y a abondance de toutes les bonnes plantes, fleurs et fruits du monde, et des arbres qu'il a pu trouver. Il fit faire les plus belles maisons et les plus beaux palais qui oncques fussent vus, car ils étaient tout dorés et décorés de toutes les belles choses du monde, et les tentures étaient toutes de soie. Il leur avait fait faire maintes charmantes fontaines, répondant aux diverses façades des palais, et toutes avaient dedans de petites conduites, où courait, en l'une vin, en d'autres lait, en d'autres miel et en d'autres l'eau la plus claire. Là habitaient les dames et damoiselles les plus belles du monde, lesquelles savaient très bien sonner de tous instruments, chanter mélodieusement, danser autour de ces fontaines mieux que toutes autres femmes, et par-

20 Le fait que le tableau représente des humains uniformément jeunes, et aucun vieillard ou enfant, donne à penser que leur génération n'est pas liée à la forme humaine de la reproduction sexuée et qu'ils vivent en dehors du temps.

21 Strubel 2013, 91.

22 Le personnage ainsi désigné par les Occidentaux de l'époque est le grand maître de la secte ismaélienne des " Assassins " qui occupaient plusieurs forteresses en Syrie et en Iran et étaient connus pour leur pratique usuelle de l'assassinat politique (voir la note de L. Hambis dans Polo 1955, 364).

23 Sourate 56, versets 14-34. Il semble que cette évocation du paradis céleste doive beaucoup à la tradition des descriptions amplifiées du jardin d'Eden. 
dessus tout, bien instruites à faire aux hommes toutes caresses et privautés imaginables. Leur rôle était d'offrir tous délices et plaisirs aux jeunes hommes qu'on mettait là ${ }^{24}$.

Les jeunes hommes, retirés de ce paradis pour accomplir les missions criminelles que leur confie le Vieux de la Montagne, obéissent avec la plus grande diligence car il leur tarde d'y revenir. L'utopie sensorielle que constitue ce pseudo paradis est donc totalement discréditée par l'auteur du récit, et cela en raison à la fois de la différence religieuse et des crimes des "Assassins ». Mais il est probable que la description de ce monde de plaisir fasse écho à celle des jardins mentionnés plus haut, disqualifiés au seul titre de leur caractère sensuel.

\section{Conclusion}

Arrivé au terme de cette étude, j'ai le sentiment d'avoir abordé beaucoup de questions disparates et présenté des suggestions dont la pertinence reste encore à établir. Pour y voir plus clair, je vais faire un inventaire des points qui me paraissent mériter une attention particulière et, pris ensemble, dessiner quelques orientations novatrices.

Le premier point est l'intérêt anthropologique de ce qu'on pourrait appeler la voie anagogique : l'accès à un absolu à travers une expérience sensorielle intense, dont le vécu effectif reste incertain, ou du moins précaire en raison de la forme humaine de la conscience du temps. D'où précisément le recours à la notion d'utopie sensorielle pour rendre compte des textes qui posent ces états comme effectivement atteints. L'hypothèse globale formulée à ce propos est la corrélation entre un apogée (quantitatif et qualitatif) de notre sensibilité et une sorte de renversement par lequel la médiation du sensible ouvre à un monde qui le transcende.

Le second point concerne les réalisations de ces états utopiques. Deux renvoient à des expériences possiblement vécues : les unes sont l'extase ou la fusion dans l'objet d'amour, telle qu'on peut en lire l'évocation dans les écrits des mystiques et la poésie de Baudelaire, les autres les états de transe ou d'extase observables à l'occasion de certains rituels. Deux autres réalisations relèvent de la spéculation théologique : l'état d'innocence heureuse de ces préhumains que sont Adam et Ève (et la foule qui peuple le "Jardin des Délices » de Bosch), et l'état de béatitude des élus - qui sont quant à eux, en tant que défunts, des post-humains. Ce qui me semble intéressant est que le vécu supposé de purs esprits soit évoqué dans le langage des sensations (surtout pour ce qui concerne les paradis « jardins des délices »), et que l'état de béatitude soit toujours associé à un dépassement du temps vers l'éternité. En somme, la catégorie de l'éternité n'apparaît pas seulement comme un attribut de la puissance divine, mais aussi comme une condition du bonheur dans ce qu'il a d'inaccessible en ce monde.

Cela conduit à une troisième hypothèse. J'ai noté en passant que les atmosphères sensorielles pourvoyeuses d'optima sensibles et de saturation sont le plus souvent le résultat de production humaines - liturgie de la cathédrale, environnement feutré de l'intimité amoureuse, etc. Or les productions de ce type, surtout lorsqu'elles sont impliquées dans des situations religieuses, sont en général des rituels. Bien des ethnographes ont noté leur richesse sensorielle, la place qu'y ont la musique, la danse, les fumigations d'aromates : autant de stimulations puissantes éventuellement capable de produire des états de transe. À cet égard, Gilbert Rouget ${ }^{25}$ a souligné que l'effet de la musique sur les états de conscience modifiée n'a rien de mécanique, 
que la transe ou d'autres états similaires sont inséparables de techniques du corps. On pourrait dire la même chose du recours à des substances psychotropes. Il est vrai, cependant, que des expériences polysensorielles intenses peuvent aussi être produites par l'ascétisme, la souffrance, la terreur $^{26}$. Dans les deux cas, c'est parce que le corps est en jeu que l'expérience vécue se modifie. La notion d'utopie sensorielle telle que je l'ai développée peut contribuer à la compréhension des situations rituelles du premier type, qui semblent offrir la réalisation approchée d'une utopie sensorielle. Dans cette perspective, il serait intéressant de développer une ethnographie de tous les aspects saillants de la polysensorialité dans les rites et de documenter les modifications qu'ils apportent au vécu du temps.

Jean-Pierre Albert EHESS, Toulouse Université de Toulouse-Jean Jaurès Maison de la recherche 5 Allées Antonio Machado 31058 Toulouse Cedex 9 Francejean-pierre.albert@ehess.fr

26 Dans le même ordre d'idée, on pourrait valider aisément l'hypothèse que les enfers et autres lieux de souffrance post mortem offrent un sensorium opposé à celui des utopies heureuses en accumulant, outre les tableaux de souffrances physiques extrêmes, les impressions négatives (vacarme, laideur, puanteur, etc.). 


\section{Bibliographie}

Albert 1990

J.-P. Albert, Odeurs de sainteté. La mythologie chrétienne des aromates, Paris 1990.

Albert 1997

J.-P. Albert, Le sang et le Ciel. Les saintes mystiques dans le monde chrétien, Paris 1997.

BAUdelaire 1961

C. Baudelaire, Les fleurs du mal, [1861], dans Euvres complètes, Paris 1961.

BeLting 2005

H. Belting, Hieronymus Bosch. Le jardin des délices, trad. française, Paris 2005.

\section{Corbin 1982}

A. Corbin, Le miasme et la jonquille. L'odorat et l'imaginaire social, $X V I I I^{e}-X I X^{e}$ siècles, Paris 1982.

Duby 1966

G. Duby, L'Europe des cathédrales, Genève 1966.

ÉPICURE 1982

Epicure, Lettres, présentation et commentaires de Jean Salem, Paris, 1982.

Hadot 1995

P. Hadot, Qu'est-ce que la philosophie antique?, Paris 1995.

Gertrude d'Helfta 1978

G. d'Helfta, Le Héraut, livre IV, Paris 1978.

\section{KANT 1978}

E. Kant, Fondements de la métaphysique des moeurs, traduction de Victor Delbos, Paris 1978.

\section{Plotin 1962}

Plotin, Ennéades, traduction d'Emile Bréhier, t. 6, $2^{\text {nde }}$ partie, Paris 1962.

Polo 1955

M. Polo, La description du monde, édition en français moderne de Louis Hambis, Paris 1955.

\section{Pseudo-Denys 1958}

Saint Denys l'Aréopagite, La hierarchie céleste, trad. de Maurice de Gandillac, Paris 1958.

\section{Rouget 1980}

G. Rouget, La musique et la transe, Paris 1980.

Strubel 2013

A. Strubel, "Le verger de déduit, un 'paradis artificiel' ? ", Cuadernos del CEMYR 21, Universidad de la Laguna, Santa Cruz de Tenerife 2013.

Saint Brendan sd. (anonyme) Le voyage de saint Brendan, ed. bilingue de Ian Short et Brian Merrilees, site saintbrendan.d-t-x.com, 22032017. 